\title{
Caregiver awareness of reproductive health issues for women with intellectual disabilities
}

\author{
Lan-Ping Lin ${ }^{1}$, Pei-Ying Lin ${ }^{2}$, Shang-Wei Hsu ${ }^{3}$, Ching-Hui Loh ${ }^{4,5}$, Jin-Ding Lin ${ }^{1,2^{*}}$, Chia-Im Lai ${ }^{6}$, \\ Wu-Chien Chien², Fu-Gong Lin²
}

\begin{abstract}
Background: Limited attention has been paid to the issue of reproductive health as it affects women with intellectual disabilities, despite reproductive health being a vital issue in public health policy for women in the general population. This paper describes caregiver awareness of reproductive health issues relative to women with intellectual disabilities who are being cared for in welfare institutions in Taiwan.

Methods: The study employed a cross-sectional, questionnaire-based study which recruited 1,152 caregivers (response rate $=71.87 \%$ ) from 32 registered disability welfare institutions in Taiwan. We classified their understanding/awareness of reproductive health issues into four domains: menstrual (1) and menopause (2) issues, sex education (3), and reproductive health services (4). Each domain had five associated yes/no questions and the total score for the four domains was out of a maximum of 20. Data were analyzed using SPSS 15.0 software.
\end{abstract}

Results: We found that most of the caregivers were familiar with matters concerning sex education, menopause, and reproductive health services, but they lacked adequate understanding of issues associated with menstruation in women with ID. Many aspects of reproductive health such as "menstrual pain", "age at menarche",

"masturbation", "diet during perimenopause", and "publicly available reproductive health services" were issues in which caregivers lacked adequate knowledge and required further instruction. Logistic regression analysis revealed that female caregivers with a university degree, and those who had experience assisting with reproductive health care were more inclined to have higher reproductive health awareness scores than their counterparts.

Conclusions: This study highlights that service providers should offer appropriate reproductive health education to institutional caregivers, and that more attention be focused on the personal experiences and concerns of intellectually disabled women in future research.

\section{Background}

There is general consensus in the healthcare community concerning the need for defined policies and services aimed specifically at reproductive health for individuals with intellectual disabilities (ID). It is likely that such undertakings would be supported by society as a whole. Previous studies have shown that the sexuality of people with ID has often been stereotyped, with this group typically characterized as being childlike and asexual, invariably leading to a denial of their socio-sexual maturity and needs. Rodgers suggested that women with ID should have more control over the non-medical

\footnotetext{
* Correspondence: a530706@ndmctsgh.edu.tw

'Graduate Institute of Life Sciences, National Defense Medical Center, Taipei, Taiwan

Full list of author information is available at the end of the article
}

aspects of menstrual management, so that their individual needs can be met [1]. Generally, people with ID have conservative attitudes towards sexual intercourse and homosexuality, but they may become involved in personal intimate contact with persons with whom they are familiar [2]. Schupf and colleagues encountered a variety of reproductive health issues, such as the ageadjusted likelihood of menopause being twice as high in women with Down syndrome as in women with other forms of ID [3], and despite the ban on involuntary sterilization, it appears that many parents and caregivers of persons with ID still support sterilization as a form of contraception, especially for persons with severe ID [2]. Servais [4] reviewed previous scientific studies that assessed the expectations and support needs of persons with ID in terms of sexual health, and pointed out that
C Biomed Central

() 2011 Lin et al; licensee BioMed Central Ltd. This is an Open Access article distributed under the terms of the Creative Commons Attribution License (http://creativecommons.org/licenses/by/2.0), which permits unrestricted use, distribution, and reproduction in any medium, provided the original work is properly cited. 
hygiene management, gynecological care, prevention of unplanned pregnancy, sexually transmitted diseases, and abuse have been frequently identified as areas in which the presence of ID dictates specific support needs.

The issue of sexuality in individuals with ID is complex and, given their cognitive limitations, frequently depends largely on the actions of caregivers [5]. Many studies have highlighted that women with ID generally receive inadequate counseling to deal with their reproductive health care, and caregivers often lacked the competence to deal with these events when they occur $[6,7]$. However, caregivers are the front-line workers for individuals with ID; they play a vital role in the provision of reproductive health care to individuals with ID, and their attitudes no doubt affect the quality of service provided to this group. McCarthy and Millard [8] suggested that research is required to establish if there are any particular aspects of appropriate reproductive health care for people with ID, particularly with respect to the attitudes of their primary caregivers. The purpose of the present study therefore is to describe caregivers' awareness of reproductive health issues with respect to women with ID who are being cared for in welfare institutions.

\section{Method}

A cross-sectional, questionnaire-based study named "Caregiver Perceptions and Health Education Strategies with respect to Menopause in Women with Intellectual Disability" was carried out. The study was given ethical approved by the Institutional Review Board of the TriService General Hospital, National Defense Medical Center (Approval number: 098-05-032). A total of 267 institutions (totaling 8508 staff members), officially registered at the end of June 2009 under the jurisdiction of the Department of Social Affairs, Ministry of the Interior in Taiwan, were included in the study $[9,10]$. The study subjects were recruited by convenience sampling, where institutions were contacted by telephone and invited to join the study. Finally, 32 institutions (16\% of the institutions contacted) agreed to participate in the study. The study population was composed of staff working in a caregiving role at these registered disability welfare institutions. Data was collected by a structured questionnaire (in Chinese) that was completed by the institutional caregivers. The survey questionnaire included an informed consent letter, the caregiver's demographic characteristics, and tested their understanding of reproductive health issues for women with ID. According to WHO Guidelines on Reproductive Health [11], reproductive health is a state of complete physical, mental and social well-being, and not merely the absence of reproductive disease or infirmity. Reproductive health deals with the reproductive processes, functions and system at all stages of life. In this study, we divided awareness of reproductive health into four domains: understanding of menstrual and menopause issues, sex education, and knowledge of reproductive health services. Each domain had five yes/ no questions, with the scoring range for each domain being 0-5 and the score for the four domains for each respondent out of a total of 20 . The questionnaire was specifically designed and, to improve its validity, was reviewed and revised by five experts in the fields of clinical medicine, public health, nursing, special education, together with welfare institute staff. Questionnaires were mailed to the institutions, and distributed to caregivers. Completed questionnaires were collected from December 22, 2009 through until February 28, 2010 inclusive. Upon receipt of the questionnaires, data were entered into a database and analyzed using SPSS 15.0 software.

\section{Results}

Of the total of 1,603 questionnaires mailed to the staff of 32 institutions, 1,152 were returned, giving a response rate of $71.87 \%$. The demographic characteristics of the respondents are shown in Table 1. Female caregivers accounted for $89.8 \%$ of the respondents. The average age of respondents was $39.77 \pm 10.23$ years (range $=$ 20-66 years), with approximately two-thirds of them possessing college and higher degrees. The respondents had spent an average of $6.62 \pm 5.97$ years (range $=$ 0.1-26 years) working in welfare disability. Most of the respondents were first-line workers, such as special educators $(47.4 \%)$ and living assistants (19.8\%).

Table 2 presents the results of caregivers' understanding of issues concerning reproductive health for women with ID in relation to menstruation, sex education, menopause, and preventive health services. In relation to their understanding of issues concerning menstruation (table 3), the mean score was $3.98 \pm 0.96$, with almost one fourth of the respondents being unfamiliar with issues concerning this domain of reproductive health $($ score $<4)$. In particular, respondents responded incorrectly to statements such as "menstrual pain is one of the symptoms of reproductive diseases" (36.7\%) and "it is abnormal to menstruate before 16 years of age" (37.1\%).

In relation to sex education for women with ID, 87.7\% of respondents had a good understanding (score $\geqq 4$ ) on the whole of issues in this domain (mean score was $4.4 \pm 0.89$ ). However, nearly one quarter of respondents (21.5\%) thought "masturbation will cause impotence or frigidity". With regard to their understanding of issues concerning menopause, most of the respondents also showed good awareness (mean score was $4.56 \pm 0.79$ ), particularly with respect to issues such as "hormonal fluctuations in the body", "menopause is a natural 
Table 1 Demographic characteristics of caregivers

\begin{tabular}{|c|c|c|c|}
\hline Variable & $\mathrm{n}$ & $\%$ & $\begin{array}{l}\text { Mean } \pm \text { SD } \\
\text { (range) }\end{array}$ \\
\hline \multicolumn{4}{|l|}{ Gender $(n=1152)$} \\
\hline Male & 117 & 10.2 & \\
\hline Female & 1035 & 89.8 & \\
\hline Age $(n=1105)$ & & & $\begin{array}{l}39.77 \pm 10.23(20- \\
66)\end{array}$ \\
\hline$<40$ & 526 & 47.6 & \\
\hline$\geqq 40$ & 579 & 52.4 & \\
\hline \multicolumn{4}{|l|}{ Educational level $(n=1134)$} \\
\hline Junior high school and less & 77 & 6.8 & \\
\hline Senior high school & 320 & 28.2 & \\
\hline College & 269 & 23.7 & \\
\hline University & 443 & 39.1 & \\
\hline Master and doctorate & 25 & 2.2 & \\
\hline \multicolumn{4}{|l|}{ Job category $(n=1120)$} \\
\hline Manager & 58 & 5.2 & \\
\hline Administrative staff & 67 & 6.0 & \\
\hline Social worker & 102 & 9.1 & \\
\hline Nurse & 42 & 3.8 & \\
\hline Special educator & 531 & 47.4 & \\
\hline Vocational trainer & 90 & 8.0 & \\
\hline Living assistant & 222 & 19.8 & \\
\hline Others & 8 & 0.7 & \\
\hline Years working in this setting $(n=983)$ & & & $6.62 \pm 5.97(0.1-26)$ \\
\hline$\leqq 5$ & 554 & 56.4 & \\
\hline $6-10$ & 196 & 19.9 & \\
\hline $11-15$ & 130 & 13.2 & \\
\hline$\geqq 16$ & 103 & 10.5 & \\
\hline $\begin{array}{l}\text { Years working in disability setting ( } \mathrm{n}= \\
\text { 991) }\end{array}$ & & & $7.32 \pm 6.29(0.1-33)$ \\
\hline$\leqq 5$ & 506 & 51.1 & \\
\hline $6-10$ & 223 & 22.5 & \\
\hline $11-15$ & 139 & 14.0 & \\
\hline$\geqq 16$ & 123 & 12.4 & \\
\hline
\end{tabular}

process and will cause risk of osteoporosis", and "women should consult with the doctor who prescribed hormone replacement medication". Only one false statement concerning "a high fat, low fiber and calcium diet are recommended during perimenopause" was incorrectly identified by $23.5 \%$ of respondents. In the final domain that dealt with "reproductive health services", the average score was $4.16 \pm 1.02$ and more than three quarters of respondents stated that they were satisfied with their awareness of issues in this domain. Nearly all of the respondents recognized the importance of breast self-examination and Pap smear tests. However, many respondents were unfamiliar with the free, publicly available reproductive health services for women in the health care system, such as a breast mammography service enabling women aged 45-69 years to undergo a free mammogram every 2 years $(29.1 \%$ of respondents
Table 2 Caregiver perceptions of reproductive health for women with ID

\begin{tabular}{ll}
\hline Items of perception & $\begin{array}{l}\text { Correct } \\
\text { responders } \\
\mathrm{n}(\%)\end{array}$
\end{tabular}

Menstrual perception

Self medication is the best way to relieve menstrual 1098 (96.0) pain $(n=1144)(R)$

Appropriate exercise is allowed during menstrual 1005 (88.4) period $(n=1137)$

The large amount of menstrual flow in the first 996 (87.4) three days is normal $(n=1140)$

It is abnormal to menstruate before 16 years of age 711 (62.9) $(\mathrm{n}=1131)$

Menstrual pain is a symptom of reproductive $\quad 716(63.3)$ diseases $(n=1131)(R)$

Sex education

It is not a crime to have sex with children under 1104 (96.4)

16 years of age $(n=1145)(R)$

It does not get pregnant to have one sex intercourse $(n=1145)(R)$

A vaginal douche after sex can avoid pregnancy $(n=1143)(R)$

Using condoms during sex intercourse can prevent getting STDs $(n=1136)$

Masturbation will cause impotence or frigidity 893 (78.5) $(\mathrm{n}=1137)(\mathrm{R})$

Menopause perception

Hormonal fluctuations will cause physical or mental issues during perimenopause $(n=1139)$

Menopause is a natural process and not a disease $(n=1146)$

Women can adjust or stop hormone replacement medication during perimenopause without consultation with their doctor $(n=1141)(R)$

Menopausal women face a high risk of osteoporosis 1057 (92.5) $(n=1143)$

A high fat, low fiber and calcium diet are

$870(76.5)$ recommended during perimenopause $(\mathrm{n}=1137)(\mathrm{R})$

Preventive health services

Monthly self breast exams are necessary to prevent cancer $(n=1147)$

Regular Pap smear tests are necessary even if you have PVC vaccinations $(n=1145)$

Regular Pap smear tests depend on the needs of women aged over 30 years $(n=1143)(R)$

There is a free breast mammography service available every 2 years for women aged $45-69$ years $(\mathrm{n}=1141)$

There is a free health exam service available every 3 years for women aged 40-64 years $(n=1133)$

Items followed by (R) are reverse scored.

unaware) and a health examination service every 3 years for adults aged $40-64$ years (32.8\% of respondents unaware). Taken together, the mean total score for the four domains was $17.08 \pm 2.51$ (range $=0-20$ ), with $31.1 \%$ of respondents scoring less than 17 points (table 4). 
Table 3 Total score of caregivers' perception by reproductive health domains

\begin{tabular}{|c|c|c|c|}
\hline Total score distribution* & $\mathrm{n}$ & $\%$ & Mean \pm SD (range) \\
\hline Menstrual perception $(n=1103)$ & & & $3.98 \pm 0.96(0-5)$ \\
\hline 0 & 10 & 0.9 & \\
\hline 1 & 13 & 1.2 & \\
\hline 2 & 46 & 4.2 & \\
\hline 3 & 202 & 18.3 & \\
\hline 4 & 480 & 43.5 & \\
\hline 5 & 352 & 31.9 & \\
\hline Sex education $(n=1126)$ & & & $4.4 \pm 0.89(0-5)$ \\
\hline 0 & 7 & 0.6 & \\
\hline 1 & 15 & 1.3 & \\
\hline 2 & 15 & 1.3 & \\
\hline 3 & 102 & 9.1 & \\
\hline 4 & 335 & 29.8 & \\
\hline 5 & 652 & 57.9 & \\
\hline Menopause perception $(n=1117)$ & & & $4.56 \pm 0.79(0-5)$ \\
\hline 0 & 7 & 0.6 & \\
\hline 1 & 7 & 0.6 & \\
\hline 2 & 13 & 1.2 & \\
\hline 3 & 61 & 5.5 & \\
\hline 4 & 265 & 23.7 & \\
\hline 5 & 764 & 68.4 & \\
\hline Reproductive health services $(n=1125)$ & & & $4.16 \pm 1.02(0-5)$ \\
\hline 0 & 5 & 0.4 & \\
\hline 1 & 11 & 1.0 & \\
\hline 2 & 65 & 5.8 & \\
\hline 3 & 195 & 17.3 & \\
\hline 4 & 291 & 25.9 & \\
\hline 5 & 558 & 49.6 & \\
\hline
\end{tabular}

*Total score: 20.

Table 5 shows the relationship between caregiver characteristics and the score received in the survey. Results of bivariate chi-square analyses showed that the respondent's gender $(\mathrm{p}=0.001)$, educational level $(\mathrm{p}=$ $0.006)$ and job category $(\mathrm{p}=0.005)$ had a statistically significant effect on reproductive health awareness scores. Table 6 shows analysis results comparing

Table 4 Total score of caregivers' reproductive health perception

\begin{tabular}{llll}
\hline Total scores & $\mathbf{n}$ & $\%$ & Mean \pm SD (range) \\
\hline Score distribution $(n=1051)$ & & & $17.08 \pm 2.51(0-20)$ \\
0 & 1 & 0.1 & \\
$1-5$ & 5 & 0.5 & \\
$6-10$ & 14 & 1.3 & \\
$11-15$ & 175 & 16.7 & \\
$16-20$ & 856 & 81.4 & \\
Score category $(n=1051)$ & & \\
$\quad<17$ & 327 & 31.1 & \\
$\geqq 17$ & 724 & 68.9 & \\
\hline
\end{tabular}

caregivers' health experience characteristics and their reproductive health awareness score, with the results showing the importance of factors such as "experience assisting with reproductive health care for women with ID" ( $p=0.005)$, "perception of adequacy" $(p<0.001)$ or "satisfaction with publicly available reproductive health services for women with ID" ( $p<0.001)$.

A multiple stepwise logistic regression was conducted to examine factors affecting the caregiver's reproductive health awareness (low and high score groups, the cut-off point score being 17 points) for women with ID. The model revealed that the factors of the caregiver's gender, educational level, and experience assisting with reproductive health care were statistically significantly associated with high reproductive health awareness for women with ID (Table 7). Those caregivers who were female $(\mathrm{OR}=1.751 ; 95 \% \mathrm{CI}=1.12-2.73)$, with a university degree $(\mathrm{OR}=1.404 ; 95 \% \mathrm{CI}=1.03-1.91)$, and those who had experience assisting with reproductive health care $(\mathrm{OR}=1.373 ; 95 \% \mathrm{CI}=1.02-1.84)$ were more inclined to have higher reproductive health awareness scores than their counterparts.

\section{Discussion}

Health issues for people with ID include respiratory problems, gastrointestinal disorders, challenging behavioral problems, and neurological conditions. This group as a whole carries a greater burden of diseases/disorders and requires more health services and preventive health interventions than the general population [12-19]. However, this group is commonly overlooked in relation to health concerns involving sexuality, sexually transmitted diseases, and end-of-life decisions [20]. Cambridge [21] suggested that the rights of people with ID to access information and receive support for sexuality and sexual health should be put first. The present paper has described caregivers' reproductive health awareness toward women with ID who are being cared for in welfare institutions. The results showed that most of the caregivers were familiar with sex education, issues of menopause, and preventive health services (mean score $\geqq 4$ ), but they were unfamiliar with issues concerning menstruation (mean score $<4$ ) in women with ID. However, many reproductive perceptions such as "menstrual pain", "age at menarche", "masturbation", "diet during perimenopause", and "free reproductive health services" were issues in which deficiencies were noted and in which caregivers needed to be provided with further instruction.

Caregivers in ID services have a clear role in encouraging women to live healthily and to ensure that women get good access to primary healthcare. Swango-Wilson [22] considered that caregivers are important to the educational experiences of individuals with ID, especially 
Table 5 Relation of caregiver characteristics and reproductive health perception score for women with ID

\begin{tabular}{|c|c|c|c|c|}
\hline \multirow[t]{2}{*}{ Variable } & \multirow[b]{2}{*}{$\mathrm{N}$} & \multicolumn{2}{|l|}{ Total score } & \multirow[b]{2}{*}{$\chi^{2}(p$ value } \\
\hline & & $<17 ;$ n (\%) & $\geqq 17 ;$ n (\%) & \\
\hline Gender & 1051 & & & 10.191 \\
\hline Male & & $50(44.2)$ & $63(55.8)$ & $(0.001)$ \\
\hline Female & & $277(29.5)$ & $661(70.5)$ & \\
\hline Age (years) & 1013 & & & 1.255 \\
\hline$<40$ & & $149(29.7)$ & $352(70.3)$ & $(0.263)$ \\
\hline$\geqq 40$ & & $169(33.0)$ & $343(67.0)$ & \\
\hline Job category & 1025 & & & 7.754 \\
\hline Front-line worker & & $228(33.8)$ & $446(66.2)$ & $(0.005)$ \\
\hline Non-front line worker & & $89(25.4)$ & $262(74.6)$ & \\
\hline Years working in current setting & 902 & & & 0.675 \\
\hline$<7$ & & $153(27.5)$ & $403(72.5)$ & $(0.411)$ \\
\hline$\geqq 7$ & & $104(30.1)$ & $242(69.9)$ & \\
\hline Years working in disability setting & 910 & & & 0.14 \\
\hline$<8$ & & $155(28.4)$ & $390(71.6)$ & $(0.708)$ \\
\hline$\geqq 8$ & & $108(29.6)$ & $257(70.4)$ & \\
\hline Marital status & 1045 & & & 3.157 \\
\hline Unmarried & & $111(33.1)$ & $224(66.9)$ & $(0.206)$ \\
\hline Married & & $184(29.1)$ & $448(70.9)$ & \\
\hline Other & & $29(37.2)$ & $49(62.8)$ & \\
\hline Educational level & 1035 & & & 7.686 \\
\hline College or less & & $201(34.4)$ & $383(65.6)$ & $(0.006)$ \\
\hline University or higher & & $119(26.4)$ & 332 (73.6) & \\
\hline Perceived health status & 1042 & & & 0.318 \\
\hline Healthy & & $12(35.3)$ & $22(64.7)$ & $(0.573)$ \\
\hline Poor & & 310 (30.8) & $698(69.2)$ & \\
\hline
\end{tabular}

Table 6 Relation of caregiver health experience characteristics and reproductive health perception score for women with ID

\begin{tabular}{|c|c|c|c|c|}
\hline \multirow[t]{2}{*}{ Variable } & \multirow[b]{2}{*}{$\mathbf{N}$} & \multicolumn{2}{|l|}{ Total score } & \multirow[b]{2}{*}{$\chi^{2}$ ( $p$ value) } \\
\hline & & $<17 ; \mathrm{n}(\%)$ & $\geqq 17 ; \mathrm{n}(\%)$ & \\
\hline Experience assisting with reproductive health care & 1028 & & & $\begin{array}{l}7.937 \\
(0.005)\end{array}$ \\
\hline Yes & & $149(27.1)$ & $400(72.9)$ & \\
\hline No & & $169(35.3)$ & $310(64.7)$ & \\
\hline Perceived adequate reproductive health knowledge & 1012 & & & $\begin{array}{l}1.133 \\
(0.287)\end{array}$ \\
\hline Inadequate & & $223(29.5)$ & $532(70.5)$ & \\
\hline Adequate & & $85(33.1)$ & $172(66.9)$ & \\
\hline Adequacy of public reproductive health services for women with ID & 1035 & & & $\begin{array}{l}15.225 \\
(<0.001)\end{array}$ \\
\hline Inadequate & & $225(28.2)$ & $572(71.8)$ & \\
\hline Adequate & & $99(41.6)$ & $139(58.4)$ & \\
\hline Satisfaction with public reproductive health services for women with ID & 1033 & & & $\begin{array}{l}14.717 \\
(<0.001)\end{array}$ \\
\hline Unsatisfied & & $171(27.0)$ & $463(73.0)$ & \\
\hline Satisfied & & $153(38.3)$ & $246(61.7)$ & \\
\hline Adequacy of institutional reproductive health services for women with ID & 1034 & & & $\begin{array}{l}1.051 \\
(0.305)\end{array}$ \\
\hline Inadequate & & $147(29.9)$ & $345(70.1)$ & \\
\hline Adequate & & $178(32.8)$ & $364(67.2)$ & \\
\hline Satisfaction with institutional reproductive health services for women with ID & 1031 & & & $\begin{array}{l}0.036 \\
(0.85)\end{array}$ \\
\hline Unsatisfied & & $89(30.8)$ & $200(69.2)$ & \\
\hline Satisfied & & $233(31.4)$ & 509 (68.6) & \\
\hline
\end{tabular}


Table 7 Logistic regression of caregiver reproductive health perception for women with ID $(\mathbf{n}=977)$ *

\begin{tabular}{|c|c|c|c|c|c|}
\hline Variable & $\beta$ & S.E. & OR & $95 \% \mathrm{Cl}$ & $p$ value \\
\hline Constant & 0.554 & 0.121 & 1.741 & & $<0.001$ \\
\hline \multicolumn{6}{|l|}{ Gender } \\
\hline Male & & & 1 & & \\
\hline Female & 0.560 & 0.226 & 1.751 & $1.12-2.73$ & 0.013 \\
\hline \multicolumn{6}{|l|}{ Job category } \\
\hline Front-line worker & & & 1 & & \\
\hline Non-front-line worker & 0.314 & 0.166 & 1.368 & $0.99-1.90$ & 0.059 \\
\hline \multicolumn{6}{|l|}{ Educational level } \\
\hline College or less & & & 1 & & \\
\hline University or higher & 0.339 & 0.156 & 1.404 & $1.03-1.91$ & 0.030 \\
\hline \multicolumn{6}{|c|}{ Experience assisting with reproductive health care for women with ID } \\
\hline No & & & 1 & & \\
\hline Yes & 0.317 & 0.151 & 1.373 & $1.02-1.84$ & 0.036 \\
\hline \multicolumn{6}{|c|}{ Adequacy of public reproductive health services for women with ID } \\
\hline Inadequate & & & 1 & & \\
\hline Adequate & -0.367 & 0.199 & 0.693 & $0.47-1.02$ & 0.065 \\
\hline \multicolumn{6}{|c|}{ Satisfaction with public reproductive health services for women with ID } \\
\hline Unsatisfied & & & 1 & & \\
\hline Satisfied & -0.263 & 0.177 & 0.768 & $0.54-1.09$ & 0.136 \\
\hline
\end{tabular}

*Divided into two groups: score $<17$ and score $\geqq 17$.

in relation to sexuality and decision-making when responding to the sexually-oriented behavior of others. Many primary caregivers believe that people with ID lack the capacity to make informed decisions about their sexuality or about having more intimate sexual relationships [23]. However, attitudes toward the sexuality of people with ID appear to have become more liberal with the passage of time. To this extent though, parents and staff differed in their attitudes, with parents holding more conservative attitudes [24]. Yool, Langdon and Garner [25] examined the attitudes of staff toward the sexuality of adults with ID within a medium-secure hospital in the United Kingdom, with their analysis revealing that staff members generally held liberal attitudes with respect to sexuality and masturbation. However, with respect to sexual intercourse, homosexual relationships, and the involvement of adults with ID in decisions regarding their own sexuality, less liberal attitudes were detected.

The attitudes of parents and teachers towards parenting by persons with ID remain negative, and these attitudes may adversely affect the provision of competency-enhancing supports and services for parents with ID and their children [2]. The present study found that the factors of institutional caregiver's gender, educational level, and experience assisting with reproductive health care were significantly associated in logistic regression analyses with high scores for reproductive health awareness for women with ID. Compared to previous studies, McConkey and Ryan [26] revealed that staff with previous experience dealing with sexual incidents involving teenagers and adults with ID, felt that they could deal with such issues more confidently in future, as did staff working in residential services rather than day services. Karellou [27] found that younger and more highly educated people expressed more contemporary attitudes towards human sexuality and the sexuality of people with ID than did older respondents. Similarly, a relationship was found between the level of education and the presence of more contemporary attitudes. Drummond [23] found primary caregivers were identified as holding more open attitudes to sexuality for the person with ID for whom they were caring, and these open attitudes were significantly influenced by a number of factors including age, level of education, marital status and religious beliefs. However, Bazzo et al. [5] found that educational level and the role of caregivers did not produce differences in their attitudes towards the sexuality of individuals with ID. A significant difference emerged between those who worked in different institutional services for this group of people.

This study has several limitations that need to be acknowledged. First, the lack of information coming directly from women with ID; our data were selfreported by key caregivers and subject to potential recall bias. In addition, the study samples were recruited from purposive sampling rather than random selection, which did not represent the full population of institutional caregivers working with ID subjects in Taiwan. 


\section{Conclusions}

The present study describes the profile of caregivers' understanding of the reproductive health of women with ID, and should provide valuable information for further educational programs to service providers. We suggest that health authorities should initiate education programs to improve the reproductive health knowledge of caregivers appropriately. Such programs need to consider factors such as the caregiver's gender, educational level, and experience assisting with reproductive health care issues, these items being significantly associated with adequate reproductive health awareness of caregivers with respect to women with ID. In addition, further research into reproductive health for women with ID is required to more precisely describe each case's personal experience in seeking care, the types of care provided, and the appropriateness of care received. To this extent, Britton [28] argued that "information giving" is not appropriate for explaining the embodied experiences of reproductive health issues such as menstruation in women with ID themselves. This approach, focusing primarily on the personal experiences and concerns of women with ID, will be necessary in future research.

\section{Acknowledgements}

This research was financially supported by National Science Council, Executive Yuan, Republic of China (Taiwan) (NSC 98-2629-B-016-001). We would like to acknowledge the contributions of the caregivers who participated in this study.

\section{Author details}

'Graduate Institute of Life Sciences, National Defense Medical Center, Taipei, Taiwan. ${ }^{2}$ School of Public Health, National Defense Medical Center, Taipei, Taiwan. ${ }^{3}$ Graduate Institute of Healthcare Administration, Asia University, Taichung, Taiwan. ${ }^{4}$ Department of Family and Community Medicine, TriService General Hospital, Taipei, Taiwan. ${ }^{5}$ Medical Affairs Bureau, Ministry of National Defense, Taipei, Taiwan. ${ }^{6}$ Office of Medical Service, Tri-Service General Hospital, National Defense Medical Center, Taipei, Taiwan.

\section{Authors' contributions}

LPL contributed to the study/questionnaire design, data collection, data analysis, and the writing of the manuscript. LPY, LCI and CWC helped the study design, data collection and the analysis of the findings. SWH, CHL and LFG participated in the study design and interpreted data. JDL had a role in conceptual framework, coordination, manuscript writing and revision. All authors read and approved the final manuscript.

\section{Competing interests}

The authors declare that they have no competing interests.

Received: 6 July 2010 Accepted: 28 January 2011

Published: 28 January 2011

\section{References}

1. Rodgers J: Pain, shame, blood, and doctors: How women with learning difficulties experience menstruation. Women's Stud Int Forum 2001, 24:523-539.

2. Aunos M, Feldman MA: Attitudes towards sexuality, sterilization and parenting rights of persons with intellectual disabilities. J Appl Res Intellect Disabil 2002, 15:285-296.
3. Schupf N, Zigman W, Kapell D, Lee JH, Kline J, Levin B: Early menopause in women with Down's syndrome. J Intellect Disabil Res 1997, 41:264-267.

4. Servais L: Sexual health care in persons with intellectual disabilities. Ment Retard Dev Disabil Res Rev 2006, 12:48-56.

5. Bazzo G, Nota L, Soresi S, Ferrari L, Minnes P: Attitudes of social service providers towards the sexuality of individuals with intellectual disability. J Appl Res Intellect Disabil 2007, 20:110-115.

6. Brown $H$, Turk V: Defining sexual abuse as it affects adults with learning disabilities. J Brit Inst Ment Handicap 1992, 20:44-55.

7. Brown H, Hunt N, Stein J: 'Alarming but very necessary': Working with staff groups around the sexual abuse of adults with learning disabilities. J Intellect Disabil Res 1994, 38:393-412.

8. McCarthy M, Millard L: Discussing the menopause with women with learning disabilities. Brit J Learn Disabil 2003, 31:9-17.

9. MOI Department of Statistics: Service workforce in disability welfare institutions in Taiwan. Taipei, Taiwan: Department of Statistics, Minitstry of the Interiors; 2009

10. MOI Department of Statistics: The service condition of institutions for the physically and mentally disabled in Taiwan. Taipei, Taiwan: Department of Statistics, Ministry of the Interiors; 2009.

11. WHO: Reproductive health indicators: Guidelines for their generation interpreation and analysis for global monitoring. WHO Geneva; 2006.

12. Lin JD, Lee TN, Loh CH, Yen CF, Hsu SW, Wu JL, Tang CC, Lin LP, Chu CM, Wu SR: Physical and mental health status of staff working for people with intellectual disabilities in Taiwan: measurement with the 36-Item Short-Form (SF-36) health survey. Res Dev Disabil 2009, 30:538-546.

13. Lin JD, Lin YW, Yen CF, Loh CH, Chwo MJ: Received, understanding and satisfaction of National Health Insurance premium subsidy scheme by families of children with disabilities: a census study in Taipei City. Res Dev Disabil 2009, 30:275-283.

14. Hsu SW, Lin YW, Chwo MJ, Huang HC, Yen CF, Lin LP, Wu JL, Lin JD: Emergency department utilization and determinants of use by 0 - to 6year-old children with disabilities in Taipei. Res Dev Disabil 2009, 30:774-781.

15. Yen CF, Lin JD, Loh CH, Shi L, Hsu SW: Determinants of prescription drug use by adolescents with intellectual disabilities in Taiwan. Res Dev Disabil 2009, 30:1354-1366.

16. Lin PY, Lin LP, Lin JD: Hypertension, hyperglycemia, and hyperlipemia among adolescents with intellectual disabilities. Res Dev Disabil 2010, 31:545-550.

17. Lin JD, Lin PY, Lin LP, Hsu SW, Loh CH, Yen CF, Fang WH, Chien WC, Tang CC, Wu CL: Prevalence and associated risk factors of anemia in children and adolescents with intellectual disabilities. Res Dev Disabil 2010, 31:25-32

18. Lin JD, Lin PY, Lin LP, Chang YY, Wu SR, Wu JL: Physical activity and its determinants among adolescents with intellectual disabilities. Res Dev Disabil 2010, 31:263-269.

19. Lin JD, Lin PY, Chen LM, Fang WH, Lin LP, Loh CH: Serum glutamicoxaloacetic transaminase (GOT) and glutamic-pyruvic transaminase (GPT) levels in children and adolescents with intellectual disabilities. Res Dev Disabil 2010, 31:172-177.

20. Prater CD, Zylstra RG: Medical care of adults with mental retardation. Am Fam Physician 2006, 73:2175-2183.

21. Cambridge P: Challenges for safer sex education and HIV prevention in services for people with intellectual disabilities in Britain. Health Promot Int 1998, 13:67-74

22. Swango-Wilson A: Caregiver perceptions and implications for sex education for individuals with intellectual and developmental disabilities. Sex Disabil 2008, 26:167-174.

23. Drummond E: Attitudes towards a pilot study in Ireland. Learn Disabil Pract 2006, 9:28-34.

24. Cuskelly M, Bryde R: Attitudes towards the sexuality of adults with an intellectual disability: Parents, support staff, and a community sample. $J$ Intellect Dev Disabil 2004, 29:255-264.

25. Yool L, Langdon PE, Garner K: The attitudes of medium-secure Unit Staff toward the sexuality of adults with learning disabilities. Sex Disabil 2003, 21:137-150.

26. McConkey R, Ryan D: Experiences of staff in dealing with client sexuality in services for teenagers and adults with intellectual disability. I Intellect Disabil Res 2001, 45:83-87. 
27. Karellou J: Laypeople's attitudes towards the sexuality of people with learning disabilities in Greece. Sex Disabil 2003, 21:65-84.

28. Britton CJ: Learning about "the curse": An anthropological perspective on experiences of menstruation. Women's Stud Int Forum 1996, 19:645-653.

Pre-publication history

The pre-publication history for this paper can be accessed here: http://www.biomedcentral.com/1471-2458/11/59/prepub

doi:10.1186/1471-2458-11-59

Cite this article as: Lin et al.: Caregiver awareness of reproductive

health issues for women with intellectual disabilities. BMC Public Health 2011 11:59

Submit your next manuscript to BioMed Central and take full advantage of:

- Convenient online submission

- Thorough peer review

- No space constraints or color figure charges

- Immediate publication on acceptance

- Inclusion in PubMed, CAS, Scopus and Google Scholar

- Research which is freely available for redistribution

Submit your manuscript at www.biomedcentral.com/submit 